\title{
Telomeres, stem cells, senescence, and cancer
}

\author{
Norman E. Sharpless ${ }^{1}$ and Ronald A. DePinho ${ }^{2}$ \\ ${ }^{1}$ Department of Medicine and Genetics, Lineberger Comprehensive Cancer Center, University of North Carolina \\ School of Medicine, Chapel Hill, North Carolina, USA \\ ${ }^{2}$ Department of Adult Oncology, Dana-Farber Cancer Institute, Department of Medicine and Genetics, \\ Harvard Medical School, Boston, Massachusetts, USA
}

\begin{abstract}
Mammalian aging occurs in part because of a decline in the restorative capacity of tissue stem cells. These self-renewing cells are rendered malignant by a small number of oncogenic mutations, and overlapping tumor suppressor mechanisms (e.g., p16 $6^{\mathrm{INK} 4 \mathrm{a}}-\mathrm{Rb}, \mathrm{ARF}-\mathrm{p} 53$, and the telomere) have evolved to ward against this possibility. These beneficial antitumor pathways, however, appear also to limit the stem cell life span, thereby contributing to aging.
\end{abstract}

J. Clin. Invest. 113:160-168 (2004). doi:10.1172/JCI200420761.

Every day, we sacrifice many varied cell types such as granulocytes, keratinocytes, hepatocytes, and erythrocytes at the altar of organismal homeostasis. For the individual to thrive, lost cells must be constantly replaced, and recent evidence has identified significant capacities for repair and regeneration even in organs once thought to be postmitotic such as the pancreatic islet and the brain. Given this continuous cellular attrition, normal tissue function requires that the rate of cell loss be matched by the rate of renewal. Aging is hastened by forces that either accelerate cellular loss or retard tissue repair. When loss exceeds repair, ensuing cellular attrition eventually leads to a decline in organ function and ultimately failure. When restricted to specific organs, this condition would be expected to result in one of the many chronic degenerative diseases such as liver cirrhosis. If, however, the process operates across multiple organ systems, then this progressive multisystem functional compromise may manifest clinically as frailty, accelerated aging, and death.

Elegant experiments from lower metazoans with postmitotic soma (e.g., Drosophila and Caenorhabditis ele-

\footnotetext{
Address correspondence to: Norman E. Sharpless, Lineberger Comprehensive Cancer Center, University of North Carolina, Chapel Hill, North Carolina 27599-7295, USA.

Phone: (919) 966-1185; Fax: (919) 966-8212;

E-mail: nes@med.unc.edu.

Conflict of interest: The authors have declared that no conflict of interest exists.

Nonstandard abbreviations used: retinoblastoma protein $(\mathrm{Rb})$; cyclin-dependent kinase inhibitor (CDKI); murine double minute 2 protein (MDM2); plasminogen activator inhibitor (PAI); senescence-associated heterochromatic foci (SAHF); telomerase RNA component (TERC); telomerase reverse transcriptase (TERT); double-strand break (DSB); telomeric repeat binding factor 2 (TRF2); alternative lengthening of telomeres (ALT); ataxia telangiectasia (AT); ataxia-telangiectasia mutated kinase (ATM); peripheral blood leukocyte (PBL); comparative genome hybridization (CGH); hematopoietic stem cell (HSC).
}

gans) have identified many of the pathways that influence the rate of cellular turnover and loss (reviewed in refs. 1, 2). These data have firmly established a link between the rate of cellular metabolism, the rate of production of unstable oxygen species, and longevity in these species. There appears little doubt that many of these conserved pathways (e.g., insulin-like growth factor-1 signaling) also influence the rate of mammalian metabolism and cellular decay, and therefore mammalian aging. As extensive tissue replacement does not occur in adults of these lower organisms, however, these model systems have been less helpful with regard to the genes that regulate tissue repair in the adult and thereby influence this aspect of aging. Therefore, one advantage of mammalian genetic systems is that they permit the investigation of the pathways responsible for the repair half of the aging equation.

To be sure, adult mammals require extensive proliferation and tissue replacement to survive. Even in the absence of pathology, the intestinal lining replaces itself entirely on a weekly basis, and the bone marrow produces trillions of new blood cells daily. An obvious cost of this massive and obligate proliferation, however, is that even under physiological conditions, it is presumed that stem cell genomes are showered with somatic mutations, some of which may target cancerrelevant genes. In accord with this view is the remarkable observation that roughly $1 \%$ of neonatal cord blood collections contain significant numbers of myeloid clones harboring oncogenic fusions such as the AML-ETO fusion associated with acute leukemia (3); similarly, as many as one in three adults possess detectable IgH-BCL2 translocations, which are commonly associated with follicular lymphoma (4). As the prevalence of these cancers is far lower in the general population, it would appear that potent tumor suppressor mechanisms function to monitor and con- 
strain the growth and survival of these aspiring cancer cells. In humans, three principal and overlapping tumor suppressor barriers appear to be operative; they are represented by the $\mathrm{p} 16^{\mathrm{INK} 4 \mathrm{a}}$-retinoblastoma protein ( $\left.\mathrm{p} 16^{\mathrm{INK} 4 \mathrm{a}}-\mathrm{Rb}\right)$ pathway, the ARF-p53 pathway, and telomeres. The combined effect of these tumor suppressor mechanisms is to place a limit on the replicative life span of cells in the compartment capable of contributing to tissue regeneration (hence termed stem cells). In this Perspective, we will discuss these tumor suppressor mechanisms and the hypothesis that their anticancer roles come at the cost of a decline in stem cell number and their proliferative reserve, thereby compromising tissue repair and promoting the aging phenotype. We will detail the human and murine data in support of this hypothesis, and discuss the implications of the intimate link between cancer and aging.

\section{Tumor suppressor pathways engender senescence and apoptosis}

A common endpoint for these major tumor suppressor mechanisms is senescence. This specialized form of terminal differentiation is induced by a variety of stimuli including alterations of telomere length and structure, some forms of DNA damage (for example, oxidative stress), and activation of certain oncogenes (reviewed in refs. 5, 6). Senescence requires activation of the $\mathrm{Rb}$ and/or $\mathrm{p} 53$ protein, and expression of their regulators such as p16 ${ }^{\mathrm{INK} 4 \mathrm{a}}, \mathrm{p} 21$, and ARF (7-10) (Figure 1). An important form of senescence is induced by p53, which has several antiproliferative activities including stimulation of the expression of $\mathrm{p} 21$, a cyclin-dependent kinase inhibitor (CDKI). CDKIs inhibit progression through the cell cycle by inhibiting cyclin-dependent kinases that phosphorylate and thereby inactivate $\mathrm{Rb}$ and related proteins $\mathrm{p} 107$ and p130 (11). The activity of p53 is predominantly mediated by inhibiting its murine double minute 2 protein (MDM2)-mediated degradation, and p53 is stabilized by diverse stimuli including DNA damage signals (e.g., resulting from oxidative stress or telomeric shortening) and oncogene activation (reviewed in refs. 12-14). The stabilization of p53 by oncogenes is in part medi- ated by ARF (also designated p19ARF in the mouse), which is induced by inappropriate cell cycle entry (15-17), and binds to MDM2, thereby inhibiting the destruction of p53 (15, 18-20). Another CDKI, $\mathrm{p} 16^{\mathrm{INK} 4 \mathrm{a}}$ increases markedly in senescent cells, and correlates with increasing $\mathrm{Rb}$ hypophosphorylation during this process $(8,9)$. The regulation of $\mathrm{p} 16^{\mathrm{INK} 4 a}$ in senescence is not as well understood as that of p53, although it appears to be induced by several stimuli including MAP kinase signaling, oncogene activation, and growth in culture (21-24). Either p53-p21 or p16 ${ }^{\text {INK4a }}$ are able to produce $\mathrm{Rb}$ hypophosphorylation and initiate senescence. Some senescence-inducing stimuli (e.g., activation of the RAS oncogene) appear to induce both pathways, while others (e.g., DNA damage) appear to preferentially activate one or the other.

Senescence differs from other physiologic forms of cell cycle arrest such as quiescence in two important ways (Table 1). First, senescence in somatic cells is generally irreversible, barring the inactivation of p53 and/or Rb, which appear to be required for its maintenance in certain settings $(10,25-27)$. Second, it is associated with distinctive molecular and morphologic alterations such as cellular flattening and increased adherence, a loss of $c$-fos induction to serum stimulation, an increased expression of plasminogen activator inhibitor (PAI) and the expression of senescence-associated $\beta$-galactosidase activity (reviewed in refs. 6,28 ). Recently, senescence has been shown to correlate with the establishment of an unusual form of heterochromatin present in discrete nuclear foci, known as senescence-associated heterochromatic foci (SAHF) (29). In aggregate, these data suggest that senescence results from the durable repression of promoters associated with growth control genes. This repression is enforced by the construction of stable, heterochromatin-like complexes, the formation of which is directed in part by hypophosphorylated $\mathrm{Rb}$.

Recent evidence suggests senescence may differ qualitatively depending on the stimulus that leads to its establishment. Several groups have shown that either $\mathrm{Rb}$ or $\mathrm{p} 53$ inactivation could reverse the senescence in murine cells or certain types of human fibroblasts

Table 1

Senescence vs. quiescence

$\begin{array}{lcc} & \text { Senescence } & \text { Quiescence } \\ \text { Stability } & \text { Permanent } & \text { Reversible } \\ \text { Induced by: } & \text { Telomere shortening } \\ & \text { Prolonged DNA damage } & \text { Serum starvation } \\ \text { Onidative stress } & \text { Growth factor deprivation } \\ \text { SA } \beta \text {-gal expression } & \text { Growth at high density } \\ \text { SA heterochromatic foci } & \text { Present } & \text { Transient DNA damage } \\ \text { Molecular markers } & \text { Present } & \text { Absent } \\ \text { Cell cycle inhibitors } & \text { Uninducible } c \text {-fos expression to serum stimulation } & \text { Absent }\end{array}$

Aln the setting of sustained $p 53$ and/or Rb function (see text for details). SA, senescence-associated; SA $\beta$-gal, senescence-associated $\beta$-galactosidase. 


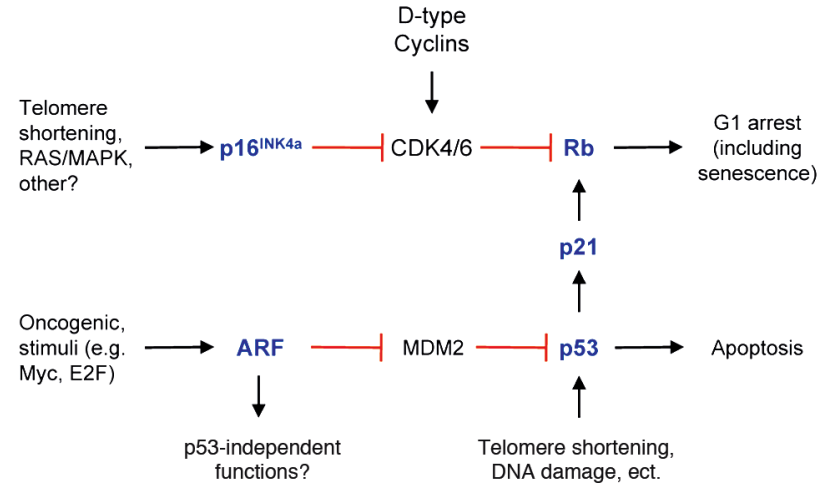

Figure 1

The p53 and Rb pathways. p53 activity is predominantly regulated at the protein level. In the unstressed state, p53 is rapidly degraded by MDM2; a process which is inhibited by ARF. Also, p53 can be stabilized by $\mathrm{N}$-terminal serine phosphorylation in response to genotoxic stresses, and this phosphorylation inhibits its interaction with MDM2. p53 activation potently induces either growth arrest or apoptosis depending on cellular context. The antiproliferative activity of p53 in part results from p 21 expression, which is a p53 transcriptional target. $\mathrm{Rb}$ is inactivated by phosphorylation as a result of the cyclin-dependent kinases CDK4 and CDK6. Hypophosphorylated $\mathrm{Rb}$ binds $\mathrm{E} 2 \mathrm{~F}$ and represses proliferation. CDK activity is inhibited by both $p 16^{\text {INK4a }}$ and $p 21$.

$(10,26,27)$. Therefore, it seems clear that persistent $\mathrm{p} 53$ and $\mathrm{Rb}$ function are required to maintain certain forms of senescence. Campisi and colleagues, however, have reported that certain human cell types senesced in a p16 $6^{\text {INK4a }}$-dependent manner, while in other cells senescence was p53-mediated (25). Intriguingly, while the authors could revert p53-mediated senescence using lentiviral vectors expressing SV40 Large T Antigen (which inactivates $\mathrm{Rb}$ and $\mathrm{p} 53$ ), $\mathrm{p} 16^{\mathrm{INK} 4 \mathrm{a}}$-mediated senescence was durable and not reversible by $\mathrm{T}$ Antigen. Of note, the formation of SAHF was more marked in p16 $6^{\text {INK4a }}$-mediated senescence, suggesting the possibility that $\mathrm{Rb}$ and $\mathrm{p} 53$ are no longer required after the establishment of these repressive chromatin structures (29). As both p16 $16^{\mathrm{INK} 4 a}$ and $\mathrm{p} 21$ induce Rb hypophosphorylation, however, the molecular basis for the difference between these forms of senescence is unclear. Therefore, robust and validated senescence markers are needed to dissect these issues.

In addition to senescence, it is worth noting that cancer-related stimuli such as oncogene activation, DNA damage, and telomere shortening can also induce an entirely distinct anticancer mechanism, namely apoptosis. Apoptotic loss of progenitor cells in response to such stimuli has been clearly demonstrated in animal models; for example, mice with shortened, dysfunctional telomeres demonstrate increased apoptosis in germ cells of the testes and crypt cells of the intestine (30-32). In these systems, an increase in apoptosis correlates with tissue atrophy and other phenotypes associated with premature aging. The role of p53 in mediating apoptosis is well-documented $(33,34)$, and this activity seems its major anticancer function in certain animal models $(35,36)$. Correspondingly, p53 loss greatly attenuates the apoptotic phenotype seen in proliferative organs in the setting of telomere dysfunction in animal models (37). While loss of p53 in these animals affords resistance to the effects of telomere dysfunction, these mice also demonstrate a marked increase in epithelial tumor formation $(37,38)$, reinforcing the view that aging and cancer are closely linked in this model system. Therefore, telomere shortening and p53 activation modulate two potent anticancer mechanisms: senescence and apoptosis. While the molecular biology of this fate-decision is incompletely understood, the specific response appears to depend on many variables including cell type, genetic context, and proliferation state. Although a role for $\mathrm{p} 16^{\mathrm{INK} 4 \mathrm{a}}$ in inducing apoptosis has been suggested (39-42), the issue of whether $\mathrm{p} 16^{\mathrm{INK} 4 \mathrm{a}}$ also has senescence-independent anticancer functions in vivo remains an area of active investigation.

\section{Telomeres, telomerase, and checkpoints}

Telomeres are nucleoprotein complexes at the chromosome ends which consist of many double-stranded TTAGGG repeats, a $3^{\prime}$ single strand overhang, and associated telomere binding proteins (Figure 2) (reviewed in refs. 43, 44). It has been known since the early days of maize genetics that telomeres play a critical role in the maintenance of chromosomal integrity - a fact that has since been confirmed in diverse model systems from yeast to plants to humans. Interestingly, however, telomere lengths differ greatly, even among mammals. For example, human telomeres are 5 to 15 $\mathrm{kb}$ long while those of inbred mice may exceed $60 \mathrm{~kb}$. Several lines of evidence have established that telomeres adopt a complex secondary and tertiary structure that relies on DNA-DNA, DNA-protein, and proteinprotein interactions. With regard to senescence, telomere structure appears at least as important to telomere function as absolute telomere length (45-47).

DNA polymerase cannot fully replicate the ends of linear DNA duplexes, and, in the absence of telomerase, chromosomes shorten slightly with every cell division. Telomerase is a specialized RNA-protein complex that is responsible for the de novo synthesis and maintenance of telomere repeats. The telomerase holoenzyme consists of a telomerase RNA component (TERC) that serves as a template for the addition of repeats, and a protein component, telomerase reverse transcriptase (TERT). Telomerase expression is low or absent in most human somatic tissues, with its expression principally restricted in the adult to activated lymphocytes, germ cells, and tissue stem cells (48-51). The restricted pattern of telomerase activity relates primarily to the strict regulation of TERT gene transcription, whereas TERC gene expression is broader in distribution. The most widely accepted model holds that progressive telomere shortening with each cell division eventually triggers an alteration in telomere structure. From the perspective 


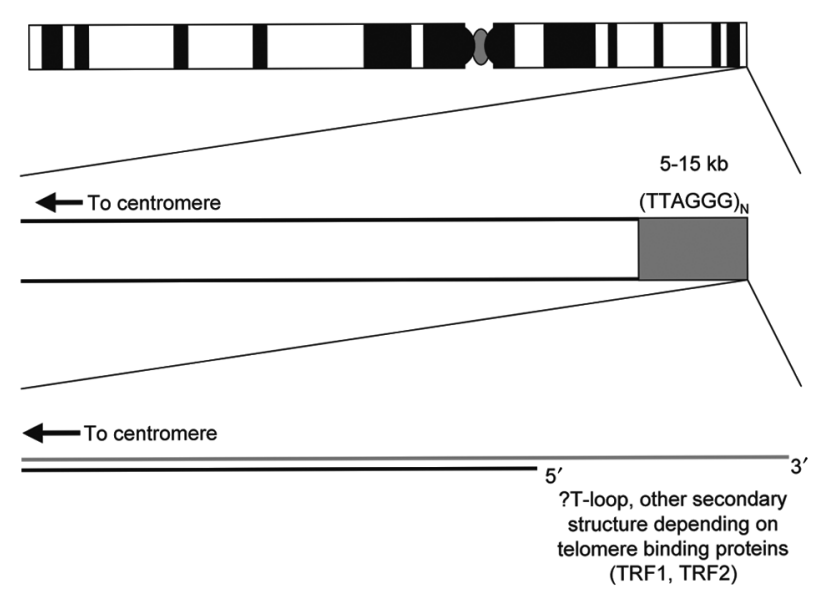

Figure 2

Telomere structure. Telomeres are present at chromosome ends. They consist of linear arrays of repeat sequences that are 5-15 kb in humans but considerably larger in mice. Telomeres also harbor a G-rich 3' overhang that is important for the adoption of proper secondary structure.

of senescence and apoptosis, the most important result of this telomere dysfunction is that the deprotected telomere end becomes, for all intents, a double-strand break (DSB) of DNA. Classical DSBs potently induce p53, and telomere dysfunction in cultured human and mouse cells has been shown to induce p53-mediated senescence or other checkpoint responses depending on the cell type.

The relationship between telomere dysfunction and activation of the $\mathrm{p} 16^{\mathrm{INK} 4 \mathrm{a}}-\mathrm{Rb}$ pathway is less clear. For example, TERT expression in cultured human fibroblasts abrogates progressive $\mathrm{p} 16^{\mathrm{INK4a}}$ accumulation and the onset of senescence (52), a result that has generally been interpreted to suggest that dysfunctional telomeres produce a signal that activates $\mathrm{p} 16^{\mathrm{INK} 4 \mathrm{a}}$. Alternatively, it is possible that TERT per se possesses functions, independent of its telomere lengthening effects, capable of quelling p $16^{\mathrm{INK} 4 \mathrm{a}}$ activation. The expression of a dominant negative form of telomeric repeat binding factor 2 (TRF2), a telomere binding protein, will induce an acute alteration in telomere structure accompanied by growth arrest in human and murine cells (45). In human cells, senescence can be induced by a dominant negative TRF2 (DN-TRF2) through either p16 ${ }^{\mathrm{INK} 4 \mathrm{a}_{-}}$or $\mathrm{p} 53$-dependent mechanisms, while, in murine cells, p53 loss alone is sufficient to abrogate growth arrest by DN-TRF2 (53). Likewise, loss of p $16^{\mathrm{INK} 4 \mathrm{a}}$ and ARF does not rescue the gonadal failure and other in vivo apoptotic responses seen in mice harboring telomere dysfunction (54). On the other hand, the placement of human epithelial cells and murine cells in culture has been shown to rapidly induce p16 $6^{\text {INK4a }}$ in the absence of telomere shortening (16, 55-57). Therefore, like $\mathrm{p} 53$, p $16^{\mathrm{INK} 4 \mathrm{a}}$ appears to be induced by telomere-dependent and -independent stimuli. The accumulation of $\mathrm{p} 16^{\mathrm{INK} 4 \mathrm{a}}$ in many tissues is noted with aging in both mice and humans $(24,58)$, but whether this results from in vivo alterations of telomere structure remains to be established. Similar$l y$, it is unclear if the major in vivo tumor suppressor function of $\mathrm{p} 16^{\mathrm{INK} 4 \mathrm{a}}$ in humans results as a response to telomere dysfunction or other stimuli.

In the absence of the p53- and p16 $6^{\text {INK4a }}$-Rb-mediated checkpoints, cultured cells with dysfunctional telomeres continue to proliferate, entering a period of slow growth called "crisis" that is characterized by genomic instability. Clones emerging from crisis invariably either reactivate telomerase (59-61) or the alternative lengthening of telomeres (ALT) mechanism $(62,63)$. During crisis, the deprotected telomere ends in proliferating cells can be illegitimately fused through DNA repair mechanisms, ultimately leading to the generation of complex non-reciprocal translocations, a hallmark feature of adult solid tumors. In a tissue stem cell, telomere dysfunction would appear to have two outcomes depending on the integrity of checkpoint mechanisms: respond to checkpoint activation with senescence/apoptosis or proliferate and engender genomic instability. Evidence suggests that most cells facing this decision respond to checkpoints, producing a progressive diminution of stem cell reserve throughout the human lifespan. This decline in tissue regeneration capacity eventually manifests as aging. The rare cells that continue to proliferate in the setting of telomere dysfunction and emerge from crisis having accumulated additional, transforming mutations are equally problematic from the organismal perspective. These cells necessarily have incurred proliferative genetic lesions and inactivated tumor suppressor checkpoints, and appear well on their way down the path to full-fledged cancer.

\section{Telomere-mediated checkpoints prevent cancer but contribute to aging}

The vast majority of cells exhibiting telomere dysfunction undergo senescence or apoptosis, and disparate lines of evidence have convincingly shown that these processes are significant barriers to cancer formation. Reactivation of telomerase is one of the most commonly observed features of cancer seen in greater than $80 \%$ of all human tumors (64-66). Telomerase expression greatly enhances the transformation of human cells in vitro (67), and tumorigenesis is inhibited by telomere shortening in animal models of cancer (54, $68,69)$. In aggregate, these data suggest that telomereinduced checkpoint activation is a major in vivo tumor suppressor mechanism.

The tumor-promoting effects of telomerase activity appear to be more complex than initially believed. Certainly, tumor clones must resolve the telomere length problem to traverse crisis, and therefore telomerase reactivation or the development of ALT is a prerequisite of malignant growth. For this reason, inhibitors of telomerase have been considered promising therapeutic candidates for novel antineoplastic agents (70). Several lines of evidence, however, also suggest that telomerase activity may contribute to tumorigenesis in a manner inde- 
pendent of its telomere lengthening effects. For example, telomerase expression has been shown, independent of telomere length, to produce resistance to the antiproliferative signals of TGF- $\beta$ in cultured mammary cells lacking p $16^{\mathrm{INK} 4 \mathrm{a}}(71)$. Additionally, animals overexpressing telomerase are more prone to neoplasia than littermate wild-type animals $(72,73)$, even though telomere length per se does not appear to limit tumor growth in these models. Lastly, transformed $\mathrm{ALT}^{+}$ murine clones demonstrate further enhanced growth in a mouse model of metastasis after transduction with telomerase (74). These disparate lines of evidence suggest that the catalytic activity of telomerase contributes to malignant growth by influencing a cellular feature in addition to absolute telomere length, but the precise mechanism of these effects has remained elusive. One possibility has been suggested by the observation that telomerase activity appears to affect the length of the telomere 3 ' overhang (Figure 2), which plays a role in assumption of proper telomere structure, thereby modulating growth arrest or apoptosis independent of absolute telomere length $(46,47)$.

In addition to preventing cancer, however, telomeremediated checkpoints appear to contribute to aging, and, as stated, mice with shortened, dysfunctional telomeres exhibit many characteristics of premature aging (31). A consideration of ataxia telangectasia (AT) is particularly intriguing in this regard. Humans with deficiency of the AT tumor suppressor (ATM) (ataxiatelangiectasia mutated kinase), which plays a role in DNA damage signaling, develop a progeroid syndrome in the setting of premature telomere shortening $(75$, 76). In comparison to humans, however, mice lacking ATM harbor a more modest phenotype in terms of aging. Such animals are markedly susceptible to thymic lymphoma (77-80), consistent with a role of ATM in sensing failed VDJ recombination intermediates (81). Strikingly, however, mice lacking ATM in the setting of telomere shortening developed accelerated aging and recapitulate more faithfully many features of the human ATM deficiency (30). Furthermore, $\mathrm{Atm}^{-1-}$ mice with short telomeres developed less thymic lymphoma despite increased mortality to non-cancer causes. Although human AT patients are tumor prone, these mice are presumably resistant to cancer because they possess an otherwise intact p53 checkpoint, and are not able to reactivate telomerase because of its germline inactivation. Therefore, many of the ageassociated phenotypes seen in AT patients appear to result from telomere dysfunction, which in turn reflects premature telomere shortening. The mechanism that produces premature telomere attrition in AT patients, however, remains unclear.

Further direct genetic evidence for a role of telomere dysfunction in human aging comes from the recent discovery that germline mutations of the telomerase complex cause the progeroid syndrome dyskeratosis congenita (82). Also, telomere shortening has been shown to precede the development of overt cirrhosis in patients with chronic hepatitis of various etiologies (83-86). In addition, several studies have demonstrated a relationship between telomere length in peripheral blood leukocytes (PBL) and the onset of certain diseases associated with aging. Such studies in nonneoplastic diseases have shown that PBL telomere lengths can provide predictive information on the risk of developing atherosclerosis $(87,88)$ and on overall mortality (89). In aggregate, these data indicate that although telomerase plays a clear role in malignant progression, telomere-induced checkpoints also contribute to certain aspects of human aging.

\section{Telomere dysfunction contributes to cancer}

As telomere-mediated checkpoints are no doubt major barriers to malignant progression in most would-be cancer cells, it therefore appears somewhat paradoxical that telomere dysfunction may also fuel tumorigenesis. Nonetheless, recent cytogenetic and molecular studies have provided strong evidence that telomere dynamics can contribute to genomic instability, particularly early in tumorigenesis. In this model, a transient period of telomere dysfunction contributes to carcinogenesis by engendering large numbers of genome-wide changes (reviewed in refs. 43, 90) (Figure 3). This occurs through breakagefusion-bridge cycles that result from the formation of dicentric chromosomes after inappropriate fusions of deprotected telomeres. This rapid reshuffling of the genetic deck produces rare cells with a threshold number of relevant changes to become full-fledged cancer.

Strong evidence that telomere-associated events are indeed relevant to carcinogenesis derives from the analysis of mice compound deficient for TERC and $p 53$ $(37,38)$. In this system, p53 deficient mice with telomere dysfunction demonstrate a marked shift in the tumor spectrum towards epithelial cancers. Additionally, these cancers exhibit the complex cytogenetic profiles found in human epithelial cancers as opposed to the more bland cytogenetic profiles of spontaneous

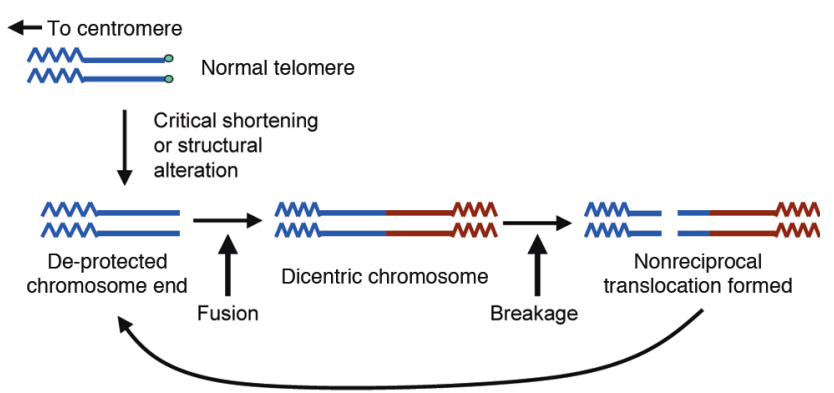

\section{Figure 3}

Bridge-fusion-breakage. Under normal circumstances, the chromosome ends are protected by telomeres. When normal cells develop telomere shortening, they undergo growth arrest or apoptosis in a p53or $\mathrm{p} 16^{1 \mathrm{NK} 4 \mathrm{a}}$-dependent manner. In cells with checkpoint inactivation, however, fusion between telomere-free ends leads to the formation of dicentric chromosomes, which are then broken during anaphase. These breakages produce non-reciprocal translocations and broken chromosome ends that are substrates for further fusion events. 


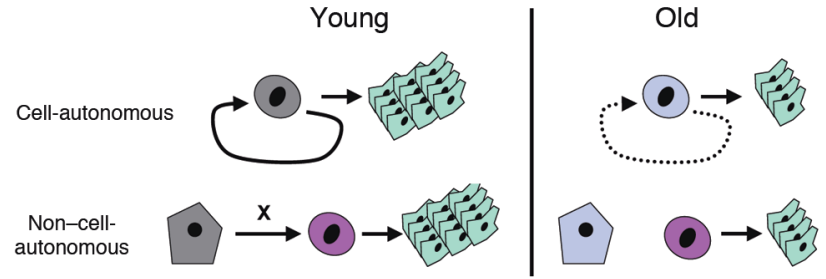

Figure 4

Cell-autonomous vs. non-cell-autonomous aging. Two models for impaired tissue repair are suggested: target cells of aging are shown in gray and senescent cell are shown in blue. In the cell-autonomous case, senescence (or apoptosis) of a progenitor with self-renewal capacity leads to impaired tissue regeneration in old animals. In the non-cell-autonomous case, however, a support cell supplies a factor $(X)$, which is critical for the maintenance of tissue repair. $X$ could be a hormone (e.g., estrogen) acting at a distance or cell-cell interactions (e.g., costimulatory signals from antigen-presenting cells) acting in a paracrine manner. In this model, aging results from the functional loss (e.g., by senescence) of the support cell, which may not be detectable in the tissue of interest.

mouse cancers $(38,91)$. While most cancers of adult humans are epithelial in origin, spontaneous carcinomas are unusual in mouse tumor models. Therefore, these data suggest that telomere dysfunction is an important event in carcinoma formation, and telomere dynamics play a major role in the species difference in carcinoma frequency between humans and mice.

This model of telomere dysfunction-driven carcinogenesis matches with the timing of telomerase activation and appearance of genomic changes during various stages of epithelial tumorigenesis. For example, comparative genome hybridization (CGH) has demonstrated that early breast (92-94) and esophageal (95, 96) lesions sustain widespread gains and losses of regions of chromosomes early in their development such ploidy changes detected by CGH correlate tightly with the presence of complex chromosomal rearrangements. This genomic instability phase is evident in stages of high-grade dysplasia, and can be found before the acquisition of a frankly malignant phenotype.

Correspondingly, the measurement of telomerase activity in corresponding preneoplastic lesions has demonstrated a consistent pattern with the ploidy and cytogenetic changes (97-103). For example, studies of adenomatous polyps and colorectal cancers have established that telomerase activity is low or undetectable in small- and intermediate-sized polyps with marked increase in telomerase activity in large adenomas and colorectal carcinomas. These data suggest that there is widespread chromosomal instability early in neoplastic progression at a time when telomerase activity is low. As these cancers progress and reactivate telomerase or ALT, genomic instability continues at a moderate rate, with further mutations presumably resulting from non-telomere-based mechanisms. Additionally, it has recently been shown that the presence of short telomeres in PBLs is associated with increased risk for the development of carcinomas of the head and neck, kidney, bladder, and lung (104). While these correlative results can be interpreted in several ways (105), substantiation of the utility of PBL telomere lengths as a reliable surrogate marker for neoplasia would prove invaluable in assessing patient risk of later developing an epithelial malignancy.

It should be noted, however, that certain cancers, particularly those malignancies with simple cytogenetics such as pediatric acute leukemia, occur without going through an obligate period of genomic instability induced by telomere dysfunction. Such tumors likely accomplish this by solving the telomere-lengthening problem prior to the onset of telomere dysfunction. Perhaps these malignancies target a telomerase-expressing compartment like the hematopoietic stem cell (HSC), or involve potent activation of oncogenes like myc that induce telomerase expression $(106,107)$. It is likely, however, that these tumors represent a special, goodprognosis case of cancer as they may neither require nor possess the large number of genomic changes seen in adult solid tumors resulting from telomere dysfunction. It is tempting to speculate that the improved prognosis and response to therapy of hematopoietic compared with epithelial cancers could relate to the fact that solid tumors experience a DNA damage phase as part of their life history. This phase may result in the obligate deactivation of DNA damage checkpoints in order to permit cancer cell survival, yet at the same time position these cancers for resistance to a large class of DNA damage-inducing cancer therapies.

\section{How are cancer and aging linked in vivo?}

The aforementioned data suggest that senescence is induced in cultured cells by a variety of telomeredependent and telomere-independent mechanisms. Likewise, the in vivo effects of inactivation (for $\mathrm{p} 53$ see ref. 108, for $\mathrm{p} 16^{\mathrm{INK} 4 \mathrm{a}}$ see refs. 109,110 , and for telomerase see refs. $21,32,111$ ) and hyperfunction (for p53 see refs. 112, 113, and for telomerase see refs. 72, 73) have been determined in mice, confirming the hypotheses that these mechanisms link cancer and aging. A problem, however, from the use of germline knockout animals and broadly expressing transgenics strains is that it is not necessarily straightforward to determine which effects are cell-autonomous and which are not (Figure 4). For example, lymphocytes from adult

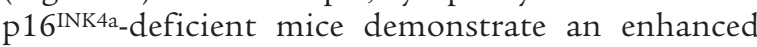
mitogenic response to CD3 and CD28 (109), and loss of this response is a hallmark feature of immunosenescence in mice (114). Problematically, however, the expression of $\mathrm{p} 16^{\mathrm{INK} 4 \mathrm{a}}$ in lymphocytes is low relative to other adult tissues (N.E. Sharpless, unpublished observation). Therefore, this resistance to aging seen in the immune system of $p 16^{I N K 4 a}$ null mice could represent a direct antiproliferative effect of $\mathrm{p} 16^{\mathrm{INK} 4 \mathrm{a}}$ on aged T-cells, or more likely could result from a role for p16 ${ }^{\text {INK4a }}$ in other cell types, such as an antigen-presenting compartment. Therefore, a future challenge will be to analyze animals with conditional inactivation of 
these pathways in order to resolve the complex effects of their loss on organismal fitness and aging.

An additional conceptual problem stems from the observation that the expression of p53 and telomere shortening do not strictly correlate with the onset of tissue aging (115-117). While undoubtedly telomere shortening is seen in certain progeroid syndromes and in states characterized by chronic hyperproliferation such as cirrhosis and myelodysplasia, it also appears that telomere length is heterogeneous in the human population, and shorter lengths do not always correlate with aging. A caveat to this conclusion, however, is that these studies necessarily have analyzed mean telomere length, although it appears that checkpoint activation may occur in response to the shortest telomeres in the cell (118). Nonetheless, while telomere shortening can be seen immediately preceding organ failure in states of high cellular turnover, it may not be an obligate feature of all normal physiologic aging. This finding points to the existence of telomere-independent causes of aging, as is almost certainly the case in normal mice.

There are two important caveats to this sort of analysis. First, only surviving cells are considered in these molecular characterizations of aged tissues. Therefore, one would not expect to detect telomere shortening and $\mathrm{p} 53$ expression in cell types where these stimuli are proapoptotic. Nonetheless, apoptotic loss of stem cells may play an important role in organismal aging. Additionally, it is possible that alterations in telomere structure, which can potently induce senescence and apoptosis (45-47), occur in vivo. Therefore, alterations in telomere dynamics may contribute to a decline in tissue function even in the absence of an overall decrease in telomere length.

Alternatively, $\mathrm{p} 53$ or $\mathrm{p} 16^{\mathrm{INK} 4 \mathrm{a}}$ activation may be contributing to aging in a very restricted compartment such as tissue stem cells. In this model, expression of p16 ${ }^{\text {INK4a }}$ or $\mathrm{p} 53$ need not be detected throughout the tissue to exert its senescence-related effects. There are data from genetic systems in support of this view. For example, loss of bmi-1, a repressor of $\mathrm{p} 16^{\mathrm{INK} 4 \mathrm{a}}$ and p19ARF, leads to a decrease in HSC number (119), and HSCs from Ink4a/Arf-deficient animals demonstrate enhanced long-term function (120). These data collectively suggest that one or both products of the Ink $4 a / A r f$ locus, both potent inducers of senescence, play a role in determining the long-term replicative potential of murine HSCs. Similarly, the effects of telomere shortening in mice are most immediately obvious in the spermatagonia and colonic crypts (32), which harbor the stem cells that give rise to the large bowel mucosa. Mice with critical telomere shortening demonstrate marked anaphase bridging and apoptosis in these progenitor-enriched compartments. Therefore, the effects of these tumor suppressor pathways may be disproportionately felt in the stem cell compartment, which represents a rare minority of cells in any given tissue.

\section{Summary}

Given the aging populations in western nations, the presently enormous problems of aging and cancer are poised to become even greater public health and socioeconomic challenges $(121,122)$. A recent study of Americans receiving Medicare support estimated the entire future lifetime health-related costs of the average healthy 70 -year-old to be approximately $\$ 150,000$ per individual (122), and the aggregate cost of this health burden will only grow larger as increasing numbers of Americans age and retire. A ray of hope from this analysis, however, was suggested by the observation that the healthiest 70-year-olds with the longest life expectancy still had the lowest future health costs. Therefore, if a goal of modern medicine is to extend patient survival, then the future solvency of Medicare appears to hinge on our ability not merely to extend overall survival, but to extend disease-free survival (123).

Our new molecular understanding of cancer and aging is a major tool in this effort, for two reasons. First, we are beginning to develop molecular surrogate markers of aging and future cancer risk. Such markers will revolutionize medical advice regarding nutrition and wellness. Amazingly, physicians have very little hard evidence to support a given dose of vitamin supplements, exercise, or diet; and most wellness therapeutics of proven benefit are directed toward a measurable predisease state like hypercholesterolemia or hypertension. By examining markers such as telomere function or $\mathrm{p} 53 / \mathrm{p} 16^{\mathrm{INK} 4 a}$ expression in a given tissue, however, we may be able to better predict the future onset of cancer and/or aging, and likewise determine the beneficial or harmful effects of a therapeutic intervention with regard to these surrogate endpoints.

Secondly, we will be able to improve our understanding of environmental or lifestyle exposures that cause or contribute to aging. Likewise, we will be able to identify which tissue compartments are targeted by these exposures, allowing a delineation of the cell-autonomous and non-cell-autonomous effects. Whether such an understanding of the environmental triggers of aging will allow us to boost the maximum human lifespan is highly controversial, but certainly at the minimum such information would help extend the healthy life expectancy; free of cancer and other adverse consequences of aging.

\section{Acknowledgments}

We thank Steven Artandi for advice and ideas regarding the manuscript. N.E. Sharpless is a Sydney Kimmel Scholar in Cancer Research and a Paul Beeson Scholar in Aging Research, and is supported by the NIH. R.A. DePinho is an American Cancer Society Professor and an Ellison Medical Foundation Senior Scholar in Aging, and is supported by the NIH.

\footnotetext{
1. Guarente, L., and Kenyon, C. 2000. Genetic pathways that regulate ageing in model organisms. Nature. 408:255-262.

2. Gems, D., and Partridge, L. 2001. Insulin/IGF signalling and ageing: seeing the bigger picture. Curr. Opin. Genet. Dev. 11:287-292.
} 
3. Mori, H., et al. 2002. Chromosome translocations and covert leukemic clones are generated during normal fetal development. Proc. Natl. Acad. Sci. U. S. A. 99:8242-8247.

4. Liu, Y., Hernandez, A.M., Shibata, D., and Cortopassi, G.A. 1994. BCL2 translocation frequency rises with age in humans. Proc. Natl. Acad. Sci. U. S. A. 91:8910-8914.

5. Wright, W.E., and Shay, J.W. 2002. Historical claims and current interpretations of replicative aging. Nat. Biotechnol. 20:682-688.

6. Campisi, J. 2001. Cellular senescence as a tumor-suppressor mechanism. Trends Cell Biol. 11:S27-S31.

7. Kamijo, T., et al. 1997. Tumor suppression at the mouse INK4a locus mediated by the alternative reading frame product p19ARF. Cell. 91:649-659

8. Stein, G.H., Drullinger, L.F., Soulard, A., and Dulic, V. 1999. Differential roles for cyclin-dependent kinase inhibitors p21 and p16 in the mechanisms of senescence and differentiation in human fibroblasts. Mol. Cell. Biol. 19:2109-2117.

9. Alcorta, D.A., et al. 1996. Involvement of the cyclin-dependent kinase inhibitor $\mathrm{p} 16$ (INK4a) in replicative senescence of normal human fibroblasts. Proc. Natl. Acad. Sci. U. S. A. 93:13742-13747.

10. Sage, J., Miller, A.L., Perez-Mancera, P.A., Wysocki, J.M., and Jacks, T. 2003. Acute mutation of retinoblastoma gene function is sufficient for cell cycle re-entry. Nature. 424:223-228.

11. Classon, M., and Harlow, E. 2002. The retinoblastoma tumour suppressor in development and cancer. Nat. Rev. Cancer. 2:910-917.

12. Vousden, K.H. 2000. p53: death star. Cell. 103:691-694.

13. Sharpless, N.E., and DePinho, R.A. 2002. p53: good cop/bad cop. Cell. 110:9-12.

14. Levine, A.J. 1997. p53, the cellular gatekeeper for growth and division. Cell. 88:323-331.

15. Pomerantz, J., et al. 1998. The Ink4a tumor suppressor gene product, p19Arf, interacts with MDM2 and neutralizes MDM2's inhibition of p53. Cell. 92:713-723.

16. Zindy, F., et al. 1998. Myc signaling via the ARF tumor suppressor regulates p53-dependent apoptosis and immortalization. Genes Dev. 12:2424-2433

17. de Stanchina, E., et al. 1998. E1A signaling to p53 involves the p19(ARF) tumor suppressor. Genes Dev. 12:2434-2442.

18. Zhang, Y., Xiong, Y., and Yarbrough, W.G. 1998. ARF promotes MDM2 degradation and stabilizes p53: ARF-INK4a locus deletion impairs both the Rb and p53 tumor suppression pathways. Cell. 92:725-734

19. Stott, F.J., et al. 1998. The alternative product from the human CDKN2A locus, p14(ARF), participates in a regulatory feedback loop with p53 and MDM2. EMBO J. 17:5001-5014.

20. Kamijo, T., et al. 1998. Functional and physical interactions of the ARF tumor suppressor with p53 and Mdm2. Proc. Natl. Acad. Sci. U. S. A. 95:8292-8297.

21. Zhu, J., Woods, D., McMahon, M., and Bishop, J.M. 1998. Senescence of human fibroblasts induced by oncogenic Raf. Genes Dev. 12:2997-3007.

22. Serrano, M., Lin, A.W., McCurrach, M.E., Beach, D., and Lowe, S.W. 1997 Oncogenic ras provokes premature cell senescence associated with accumulation of p53 and p16INK4a. Cell. 88:593-602.

23. Lin, A.W., et al. 1998. Premature senescence involving p53 and p16 is activated in response to constitutive MEK/MAPK mitogenic signaling. Genes Dev. 12:3008-3019.

24. Zindy, F., Quelle, D.E., Roussel, M.F., and Sherr, C.J. 1997. Expression of the p16INK4a tumor suppressor versus other INK4 family members during mouse development and aging. Oncogene. 15:203-211.

25. Beausejour, C.M., et al. 2003. Reversal of human cellular senescence: Roles of the p53 and p16 pathways. EMBO J. 22:4212-4222.

26. Dirac, A.M., and Bernards, R. 2003. Reversal of senescence in mouse fibroblasts through lentiviral suppression of p53. J. Biol. Chem. 278:11731-11734.

27. Gire, V., and Wynford-Thomas, D. 1998. Reinitiation of DNA synthesis and cell division in senescent human fibroblasts by microinjection of anti-p53 antibodies. Mol. Cell. Biol. 18:1611-1621.

28. Sharpless, N.E. 2003. The persistence of senescence. Sci. Aging Knowledge Environ. 2003:PE24.

29. Narita, M., et al. 2003. Rb-mediated heterochromatin formation and silencing of E2F target genes during cellular senescence. Cell. 113:703-716.

30. Wong, K.K., et al. 2003. Telomere dysfunction and Atm deficiency compromises organ homeostasis and accelerates ageing. Nature. 421:643-648.

31. Rudolph, K.L., et al. 1999. Longevity, stress response, and cancer in aging telomerase-deficient mice. Cell. 96:701-712.

32. Lee, H.W., et al. 1998. Essential role of mouse telomerase in highly proliferative organs. Nature. 392:569-574

33. Lowe, S.W., Ruley, H.E., Jacks, T., and Housman, D.E. 1993. p53-dependent apoptosis modulates the cytotoxicity of anticancer agents. Cell. 74:957-967.

34. Yonish-Rouach, E., et al. 1991. Wild-type p53 induces apoptosis of myeloid leukaemic cells that is inhibited by interleukin-6. Nature. 352:345-347.
35. Schmitt, C.A., et al. 2002. Dissecting p53 tumor suppressor functions in vivo. Cancer Cell. 1:289-298.

36. Lu, X., et al. 2001. Selective inactivation of p53 facilitates mouse epithelial tumor progression without chromosomal instability. Mol. Cell. Biol. 21:6017-6030.

37. Chin, L., et al. 1999. p53 deficiency rescues the adverse effects of telomere loss and cooperates with telomere dysfunction to accelerate carcinogenesis. Cell. 97:527-538.

38. Artandi, S.E., et al. 2000. Telomere dysfunction promotes non-reciprocal translocations and epithelial cancers in mice. Nature. 406:641-645.

39. Ausserlechner, M.J., et al. 2001. The cell cycle inhibitor p16(INK4A) sensitizes lymphoblastic leukemia cells to apoptosis by physiologic glucocorticoid levels. J. Biol. Chem. 276:10984-10989.

40. Lagresle, C., et al. 2002. Transgenic expression of the p16(INK4a) cyclindependent kinase inhibitor leads to enhanced apoptosis and differentiation arrest of CD4-CD8-immature thymocytes. J. Immunol. 168:2325-2331.

41. Plath, T., et al. 2000. A novel function for the tumor suppressor p16(INK4a): induction of anoikis via upregulation of the alpha(5)beta(1) fibronectin receptor. J. Cell Biol. 150:1467-1478.

42. Tamm, I., et al. 2002. Adenovirus-mediated gene transfer of P16INK4/CDKN2 into bax-negative colon cancer cells induces apoptosis and tumor regression in vivo. Cancer Gene Ther. 9:641-650.

43. Maser, R.S., and DePinho, R.A. 2002. Connecting chromosomes, crisis, and cancer. Science. 297:565-569.

44. de Lange, T. 2002. Protection of mammalian telomeres. Oncogene. 21:532-540.

45. van Steensel, B., Smogorzewska, A., and de Lange, T. 1998. TRF2 protects human telomeres from end-to-end fusions. Cell. 92:401-413.

46. Stewart, S.A., et al. 2003. Erosion of the telomeric single-strand overhang at replicative senescence. Nat. Genet. 33:492-496.

47. Masutomi, K., et al. 2003. Telomerase maintains telomere structure in normal human cells. Cell. 114:241-253.

48. Broccoli, D., Young, J.W., and de Lange, T. 1995. Telomerase activity in normal and malignant hematopoietic cells. Proc. Natl. Acad. Sci. U. S. A 92:9082-9086.

49. Counter, C.M., Gupta, J., Harley, C.B., Leber, B., and Bacchetti, S. 1995 Telomerase activity in normal leukocytes and in hematologic malignancies. Blood. 85:2315-2320.

50. Wright, W.E., Piatyszek, M.A., Rainey, W.E., Byrd, W., and Shay, J.W. 1996. Telomerase activity in human germline and embryonic tissues and cells. Dev. Genet. 18:173-179.

51. Weng, N.P., Levine, B.L., June, C.H., and Hodes, R.J. 1996. Regulated expression of telomerase activity in human $\mathrm{T}$ lymphocyte development and activation. J. Exp. Med. 183:2471-2479.

52. Bodnar, A.G., et al. 1998. Extension of life-span by introduction of telomerase into normal human cells. Science. 279:349-352.

53. Smogorzewska, A., and de Lange, T. 2002. Different telomere damage signaling pathways in human and mouse cells. EMBO J. 21:4338-4348.

54. Greenberg, R.A., et al. 1999. Short dysfunctional telomeres impair tumorigenesis in the INK4a(delta2/3) cancer-prone mouse. Cell. 97:515-525.

55. Foster, S.A., Wong, D.J., Barrett, M.T., and Galloway, D.A. 1998. Inactivation of 16 in human mammary epithelial cells by CpG island methylation. Mol. Cell. Biol.18:1793-1801.

56. Huschtscha, L.I., et al. 1998. Loss of p16INK4 expression by methylation is associated with lifespan extension of human mammary epithelial cells Cancer Res. 58:3508-3512.

57. Kiyono, T., et al. 1998. Both Rb/p16INK4a inactivation and telomerase activity are required to immortalize human epithelial cells. Nature. 396:84-88

58. Nielsen, G.P., et al. 1999. Immunohistochemical survey of p16INK4A expression in normal human adult and infant tissues. Lab. Invest. 79:1137-1143.

59. Counter, C.M., et al. 1992. Telomere shortening associated with chromosome instability is arrested in immortal cells which express telomerase activity. EMBO J. 11:1921-1929.

60. Coursen, J.D., Bennett, W.P., Gollahon, L., Shay, J.W., and Harris, C.C. 1997. Genomic instability and telomerase activity in human bronchial epithelial cells during immortalization by human papillomavirus-16 E6 and E7 genes. Exp. Cell Res. 235:245-253.

61. Counter, C.M., Botelho, F.M., Wang, P., Harley, C.B., and Bacchetti, S 1994. Stabilization of short telomeres and telomerase activity accompany immortalization of Epstein-Barr virus-transformed human B lymphocytes. J. Virol. 68:3410-3414

62. Bryan, T.M., Englezou, A., Gupta, J., Bacchetti, S., and Reddel, R.R. 1995 Telomere elongation in immortal human cells without detectable telomerase activity. EMBO J. 14:4240-4248.

63. Bryan, T.M., Marusic, L., Bacchetti, S., Namba, M., and Reddel, R.R. 1997. The telomere lengthening mechanism in telomerase-negative immortal human cells does not involve the telomerase RNA subunit. Hum. Mol Genet. 6:921-926.

64. Hiyama, K., et al. 1995. Telomerase activity in small-cell and non-smallcell lung cancers. J. Natl. Cancer Inst. 87:895-902. 
65. Kim, N.W., et al. 1994. Specific association of human telomerase activity with immortal cells and cancer. Science. 266:2011-2015.

66. Hiyama, E., et al. 1995. Correlating telomerase activity levels with human neuroblastoma outcomes. Nat. Med. 1:249-255.

67. Hahn, W.C., et al. 1999. Creation of human tumour cells with defined genetic elements. Nature. 400:464-468.

68. Gonzalez-Suarez, E., Samper, E., Flores, J.M., and Blasco, M.A. 2000. Telomerase-deficient mice with short telomeres are resistant to skin tumorigenesis. Nat. Genet. 26:114-117.

69. Rudolph, K.L., Millard, M., Bosenberg, M.W., and DePinho, R.A. 2001. Telomere dysfunction and evolution of intestinal carcinoma in mice and humans. Nat. Genet. 28:155-159.

70. Shay, J.W., and Wright, W.E. 2002. Telomerase: a target for cancer therapeutics. Cancer Cell. 2:257-265.

71. Stampfer, M.R., et al. 2001. Expression of the telomerase catalytic subunit, hTERT, induces resistance to transforming growth factor beta growth inhibition in p16INK4A(-) human mammary epithelial cells. Proc. Natl. Acad. Sci. U. S. A. 98:4498-4503.

72. Artandi, S.E., et al. 2002. Constitutive telomerase expression promotes mammary carcinomas in aging mice. Proc. Natl. Acad. Sci. U. S. A 99:8191-8196.

73. Gonzalez-Suarez, E., et al. 2001. Increased epidermal tumors and increased skin wound healing in transgenic mice overexpressing the catalytic subunit of telomerase, mTERT, in basal keratinocytes. EMBO J. 20:2619-2630

74. Chang, S., Khoo, C.M., Naylor, M.L., Maser, R.S., and DePinho, R.A. 2003. Telomere-based crisis: functional differences between telomerase activation and ALT in tumor progression. Genes Dev. 17:88-100.

75. Metcalfe, J.A., et al. 1996. Accelerated telomere shortening in ataxia telangiectasia. Nat. Genet. 13:350-353.

76. Xia, S.J., Shammas, M.A., and Shmookler Reis, R.J. 1996. Reduced telomere length in ataxia-telangiectasia fibroblasts. Mutat. Res. 364:1-11.

77. Borghesani, P.R., et al. 2000. Abnormal development of Purkinje cells and lymphocytes in Atm mutant mice. Proc. Natl. Acad. Sci. U. S. A. 97:3336-3341.

78. Barlow, C., et al. 1996. Atm-deficient mice: a paradigm of ataxia telangiectasia. Cell. 86:159-171.

79. Xu, Y., et al. 1996. Targeted disruption of ATM leads to growth retardation, chromosomal fragmentation during meiosis, immune defects, and thymic lymphoma. Genes Dev. 10:2411-2422.

80. Elson, A., et al. 1996. Pleiotropic defects in ataxia-telangiectasia proteindeficient mice. Proc. Natl. Acad. Sci. U. S. A. 93:13084-13089.

81. Liao, M.J., and Van Dyke, T. 1999. Critical role for Atm in suppressing V(D)J recombination-driven thymic lymphoma. Genes Dev. 13:1246-1250.

82. Vulliamy, T., et al. 2001. The RNA component of telomerase is mutated in autosomal dominant dyskeratosis congenita. Nature. 413:432-435.

83. Kitada, T., Seki, S., Kawakita, N., Kuroki, T., and Monna, T. 1995. Telomere shortening in chronic liver diseases. Biochem. Biophys. Res. Commun. 211:33-39.

84. Miura, N., et al. 1997. Progressive telomere shortening and telomerase reactivation during hepatocellular carcinogenesis. Cancer Genet. Cytogenet. 93:56-62.

85. Urabe, Y., et al. 1996. Telomere length in human liver diseases. Liver. 16:293-297.

86. Wiemann, S.U., et al. 2002. Hepatocyte telomere shortening and senescence are general markers of human liver cirrhosis. FASEB J. 16:935-942.

87. Samani, N.J., Boultby, R., Butler, R., Thompson, J.R., and Goodall, A.H. 2001. Telomere shortening in atherosclerosis. Lancet. 358:472-473.

88. Obana, N., et al. 2003. Telomere shortening of peripheral blood mononuclear cells in coronary disease patients with metabolic disorders. Intern. Med. 42:150-153.

89. Cawthon, R.M., Smith, K.R., O’Brien, E., Sivatchenko, A., and Kerber, R.A. 2003. Association between telomere length in blood and mortality in people aged 60 years or older. Lancet. 361:393-395.

90. Artandi, S.E., and DePinho, R.A. 2000. A critical role for telomeres in suppressing and facilitating carcinogenesis. Curr. Opin. Genet. Dev. 10:39-46.

91. O’Hagan, R.C., et al. 2002. Telomere dysfunction provokes regional amplification and deletion in cancer genomes. Cancer Cell. 2:149-155.

92. Buerger, H., et al. 1999. Comparative genomic hybridization of ductal carcinoma in situ of the breast-evidence of multiple genetic pathways. J. Pathol. 187:396-402.

93. Waldman, F.M., et al. 2000. Chromosomal alterations in ductal carcinomas in situ and their in situ recurrences. J. Natl. Cancer Inst. 92:313-320.

94. Moore, E., Magee, H., Coyne, J., Gorey, T., and Dervan, P.A. 1999. Widespread chromosomal abnormalities in high-grade ductal carcinoma in situ of the breast. Comparative genomic hybridization study of pure high-grade DCIS. J. Pathol. 187:403-409.

95. Walch, A.K., et al. 2000. Chromosomal imbalances in Barrett's adenocarcinoma and the metaplasia-dysplasia-carcinoma sequence. Am. J. Pathol. 156:555-566.

96. van Dekken, H., Vissers, C.J., Tilanus, H.W., Tanke, H.J., and Rosenberg C. 1999. Clonal analysis of a case of multifocal oesophageal (Barrett's) adenocarcinoma by comparative genomic hybridization. J. Pathol. 188:263-266.

97. Lord, R.V., et al. 2000. Telomerase reverse transcriptase expression is increased early in the Barrett's metaplasia, dysplasia, adenocarcinoma sequence. J. Gastrointest. Surg. 4:135-142.

98. Chadeneau, C., Hay, K., Hirte, H.W., Gallinger, S., and Bacchetti, S. 1995 Telomerase activity associated with acquisition of malignancy in human colorectal cancer. Cancer Res. 55:2533-2536.

99. Engelhardt, M., Drullinsky, P., Guillem, J., and Moore, M.A. 1997. Telomerase and telomere length in the development and progression of premalignant lesions to colorectal cancer. Clin. Cancer Res. 3:1931-1941.

100.Yan, P., Saraga, E.P., Bouzourene, H., Bosman, F.T., and Benhattar, J. 1999. Telomerase activation in colorectal carcinogenesis. J. Pathol. 189:207-212.

101.Tang, R., Cheng, A.J., Wang, J.Y., and Wang, T.C. 1998. Close correlation between telomerase expression and adenomatous polyp progression in multistep colorectal carcinogenesis. Cancer Res. 58:4052-4054.

102.Poremba, C., et al. 1998. Telomerase activity in human proliferative breast lesions. Int. J. Oncol. 12:641-648.

103.Tsao, J., et al. 1997. Telomerase activity in normal and neoplastic breast. Clin. Cancer Res. 3:627-631.

104. Wu, X., et al. 2003. Telomere dysfunction: a potential cancer predisposition factor. J. Natl. Cancer Inst. 95:1211-1218.

105.Wong, K.K., and DePinho, R.A. 2003. Walking the telomere plank into cancer. J. Natl. Cancer Inst. 95:1184-1186.

106. Greenberg, R.A., et al. 1999. Telomerase reverse transcriptase gene is a direct target of c-Myc but is not functionally equivalent in cellular transformation. Oncogene. 18:1219-1226.

107. Wang, J., Xie, L.Y., Allan, S., Beach, D., and Hannon, G.J. 1998. Myc activates telomerase. Genes Dev. 12:1769-1774.

108. Donehower, L.A., et al. 1992. Mice deficient for p53 are developmentally normal but susceptible to spontaneous tumours. Nature. 356:215-221.

109.Sharpless, N.E., et al. 2001. Loss of p16Ink4a with retention of p19Arf predisposes mice to tumorigenesis. Nature. 413:86-91.

110.Krimpenfort, P., Quon, K.C., Mooi, W.J., Loonstra, A., and Berns, A. 2001 Loss of p16Ink4a confers susceptibility to metastatic melanoma in mice. Nature. 413:83-86.

111. Blasco, M.A., et al. 1997. Telomere shortening and tumor formation by mouse cells lacking telomerase RNA. Cell. 91:25-34.

112.Tyner, S.D., et al. 2002. p53 mutant mice that display early ageing-associated phenotypes. Nature. 415:45-53.

113. Garcia-Cao, I., et al. 2002. "Super p53" mice exhibit enhanced DNA damage response, are tumor resistant and age normally. EMBO J. 21:6225-6235.

114.Engwerda, C.R., Handwerger, B.S., and Fox, B.S. 1994. Aged T cells are hyporesponsive to costimulation mediated by CD28. J. Immunol. 152:3740-3747.

115. Hastie, N.D., et al. 1990. Telomere reduction in human colorectal carcinoma and with ageing. Nature. 346:866-868.

116.Allsopp, R.C., et al. 1992. Telomere length predicts replicative capacity of human fibroblasts. Proc. Natl. Acad. Sci. U. S. A. 89:10114-10118.

117. Frenck, R.W., Jr., Blackburn, E.H., and Shannon, K.M. 1998. The rate of telomere sequence loss in human leukocytes varies with age. Proc. Natl. Acad. Sci. U. S. A. 95:5607-5610.

118.Hemann, M.T., Strong, M.A., Hao, L.Y., and Greider, C.W. 2001. The shortest telomere, not average telomere length, is critical for cell viability and chromosome stability. Cell. 107:67-77.

119.Park, I.K., et al. 2003. Bmi-1 is required for maintenance of adult selfrenewing haematopoietic stem cells. Nature. 423:302-305.

120.Lewis, J.L., et al. 2001. The influence of INK4 proteins on growth and selfrenewal kinetics of hematopoietic progenitor cells. Blood. 97:2604-2610.

121.Spillman, B.C., and Lubitz, J. 2000. The effect of longevity on spending for acute and long-term care. N. Engl. J. Med. 342:1409-1415.

122.Lubitz, J., Cai, L., Kramarow, E., and Lentzner, H. 2003. Health, life expectancy, and health care spending among the elderly. N. Engl.J. Med. 349:1048-1055.

123.Cutler, D.M. 2003. Disability and the future of Medicare. N. Engl. J. Med. 349:1084-1085. 